\title{
A Review of Performance Analyzing on Public and Private Blockchain Platforms
}

\author{
Muhammad Firdaus (201956717) \\ Department of Information Security, Graduate School \\ Pukyong National University \\ e-mail: mfirdaus@pukyong.ac.kr
}

\begin{abstract}
Summary: Blockchain is a promising new technology, generating widespread interest, and receiving considerable attention in the research community, such as academia and industry. This interest started with the success of Bitcoin but took speed with the promise of smart contracts and a vast number of applications. While there is a broad interest in developing blockchain systems for specific use cases, there is a lack of tools to perform their evaluation and implementation decisions may hamper fast progress. This report provides the review of security and performance of public and private blockchain framework, where each of which is represented by the two well-known papers, the first is titled "On the Security and Performance of Proof of Work Blockchains" and the second paper is titled "BLOCKBENCH: A Framework for Analyzing Private Blockchains". The first paper introduces a novel quantitative framework to analyze the security and performance implications of various consensus and network parameters of PoW blockchains. The framework allows for capture existing PoW-based deployments as well as PoW blockchain variants that are instantiated with different parameters, and to objectively compare the tradeoffs between their performance and security provisions. In the second paper, the authors describe BlockBench, the first evaluation framework for analyzing private blockchains. BlockBench measures overall and componentwise performance in terms of throughput, latency, scalability, and fault-tolerance. Next, BlockBench is used to conduct a comprehensive evaluation of three major private blockchains: Ethereum, Parity, and Hyperledger Fabric. Furthermore, there are gaps in performance among the three systems which are attributed to the design choices at different layers of the blockchain's software stack.
\end{abstract}

\section{INTRODUCTION}

Blockchain technologies are gaining massive momentum in the last few years, since its inception in 2009, Bitcoin's blockchain has fueled innovation and a number of novel applications, such as smart contracts, have been designed to take advantage of the blockchain. Bitcoin has been forked a number of times in order to fine-tune the consensus (i.e., the block generation time and the hash function), and the network parameters (e.g., the size of blocks and the information propagation protocol) and to increase the blockchain's efficiency. Although a number of consensus protocols (PBFT, Proof of Stack, Proof of Elapsed Time) have been proposed, most existing blockchain leverage the computationally expensive Proof of Work (PoW) consensus mechanism. However, the relationship between performance and security provisions of PoW blockchains has so far not been studied in much detail.

Interest from the industry has started to drive development of new blockchain platforms that are designed for private settings in which participants are authenticated. Blockchain systems in such environments are called private (or permissioned), as 
opposed to the early systems operating in public (or permissionless) environments where anyone can join and leave. These systems target and aim to disrupt applications which have so far been implemented on top of database systems, for example banking, finance and trading applications. Multiple platforms for private blockchains are being actively developed and fine tuned. However, there is a clear lack of a systematic framework with which different systems can be analyzed and compared against each other.

This report provides the review of security and performance of public and private blockchain framework, where each of which is represented by the two well-known papers, the first paper [1] written by Gervais et al. introduces a novel quantitative framework to analyze the security and performance implications of various consensus and network parameters of PoW blockchains, and the second paper [2] written by Dinh et al. describe BlockBench, the first evaluation framework for analyzing private blockchains. In [1], the framework allows for capture existing PoW-based deployments as well as PoW blockchain variants that are instantiated with different parameters, and to objectively compare the tradeoffs between their performance and security provisions. In [2], BlockBench measures overall and componentwise performance in terms of throughput, latency, scalability, and fault-tolerance. Further, BlockBench is used to conduct a comprehensive evaluation of three major private blockchains: Ethereum, Parity, and Hyperledger Fabric.

\section{PUBLIC BLOCKCHAIN PLATFORMS}

In the original design, Bitcoin's blockchain use a Proof of Work (PoW) system to implement a distributed timestamp server on a peer-to-peer basis. Bitcoin employs a hash-based PoW which entails finding a nonce value, such that when hashed with additional block parameters, the value of the hash has to be smaller than the current target value. When such a nonce is found, the miner creates the block and forwards it on the network layer to its peers. Other peers in the network can verify the PoW by computing the hash of the block and checking whether it satisfies the condition to be smaller than the current target value.

PoW's security relies on the principle that no entity should gather more than $50 \%$ of the processing power because such an entity can effectively control the system by sustaining the longest chain. Here, there are two known attacks on existing PoW-based blockchains. First, an adversary can attempt to double-spend by using the same coin(s) to issue two (or more) transactions, thus effectively spending more coins than he possesses. Second, miners might attempt to perform selfish mining attacks in order to increase their relative mining share in the blockchain, by selectively withholding mined blocks and only gradually publishing them.

\subsection{Contributions of The Paper}

Below are contributions of the paper [1]: 
- Quantitative framework

To analyse the security and performance implications of various consensus and network parameters of PoW blockchains. Leveraging the framework [1], Gervais et al. capture the security properties of existing PoW instantiations (e.g., Bitcoin, Ethereum, Litecoin, and Dogecoin) as well as other possible instantiations subject to different consensus and network parameters. Further, the framework allows to compare security of PoW blockchain, such as two main attacks that are relevant, double spending and selfish mining.

- Develop an open-source blockchain simulator

To evaluate the security and performance of a number of variant PoW-based blockchain instances. Based on the framework [1], Gervais et al. devise optimal adversarial strategies for doublespending and selfish mining while taking into account real world constraints such as network propagation, different block sizes, block generation intervals, information propagation mechanism, and the impact of eclipse attacks.

Figure 1 shows the framework of [1], consists of two key elements: (i) a blockchain instance and (ii) a blockchain security model. A blockchain instance is a PoW blockchain instantiated with a given set of consensus and network parameters, such as network delays, block generation times, block sizes, information propagation mechanisms, etc. For example, Bitcoin, Litecoin, and Ethereum correspond to 3 different blockchain instances.

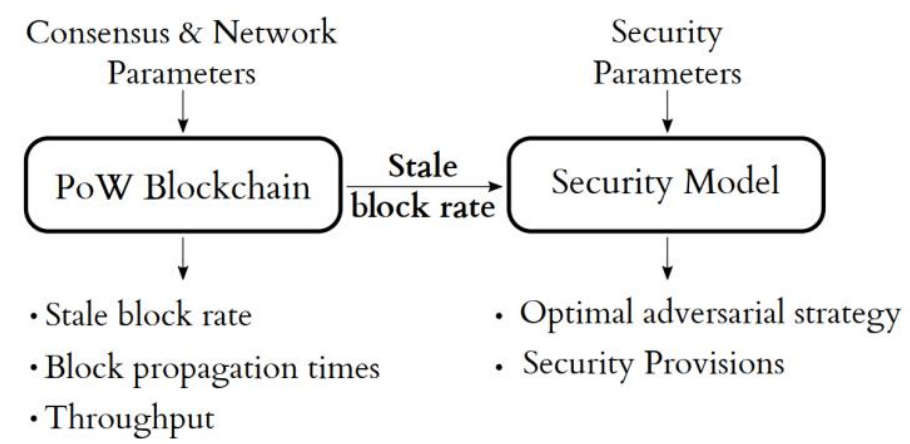

Figure 1. Components of quatitative framework

The main output of the blockchain instance is the stale block rate, which is fed as input into our security model. On the other hand, the security model is based on Markov Decision Processes (MDP) for double-spending and selfish mining and allows the reason about optimal adversarial strategies while taking into account the adversarial mining power, the impact of eclipse attacks, block rewards, and real world network and consensus parameters. 


\subsection{Bitcoin Simulator}

Bitcoin Simulator is built on ns3, the popular discrete-event simulator. They [1] use of rapidjson to facilitate the communication process among the nodes. The purpose of this simulator is to study how consensus parameteres, network characteristics and protocol modifications affect the scalability, security and efficiency of Proof of Work powered blockchains. By leveraging simulator, we can evaluate different blockchain parameters, such as the block interval, the block size, the propagation mechanisms by measuring the resulting stale block rate, throughput and block propagation times. They feed the stale block rate output by the simulator into MDP model in order to assess the security (under selfish mining and double-spending) of the resulting blockchain instance. In this simulator, there are two different node type: (i) regular nodes, and (ii) miners. Table 1 summarize the parameters captured by simulator.

\begin{tabular}{ll}
\hline Consensus parameter & Description \\
\hline Block interval distribution & Time to find a block \\
Mining power distribution of the miners & PoW power distribution \\
\hline Network-layer parameter & Description \\
\hline Block size distribution & Variable transaction load \\
\# of reachable network nodes & Open TCP port nodes \\
Geo. distribution of nodes & Worldwide distribution \\
Geo. mining pool distribution & Worldwide distribution \\
\# of connections per node & Within network \\
\# of connections of the miners & Within network \\
Block request management system & Possible Protocols \\
\hline Standard mechanism (inv/getdata) & Default \\
Unsolicited block push & Miner only push block \\
Relay network & Miner network \\
Sendheaders & Bitcoin v0.12 \\
\hline
\end{tabular}

Table 1. Parameters of the blockchain simulation

\subsection{Performance Analysis}

Here, the experimental result of bitcoin simulator:

- Simulator validation

The results show that the measured and simulated median block propagation times are relatively close. The stale block rates for Litecoin and Dogecoin are particularly close. In the case of Bitcoin, the stale rate falls between the case when all miners use the relay network and unsolicited block push, and the extreme case where the relay network and unsolicited block push is not used by any miner. 


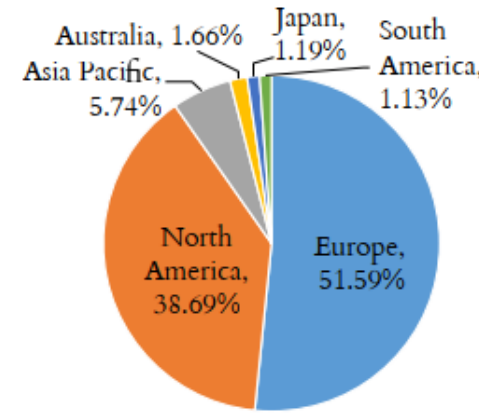

(a) Node distribution.

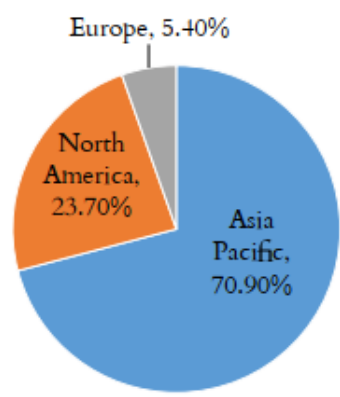

(b) Miner distribution.

Figure 2. Geo Geographical distribution of Bitcoin nodes and miners used in bitcoin simulator

\begin{tabular}{llll}
\hline & Bitcoin & Litecoin & Dogecoin \\
\hline Block interval & $10 \mathrm{~min}$ & $2.5 \mathrm{~min}$ & $1 \mathrm{~min}$ \\
\hline Measured $t_{M B P}$ & $8.7 \mathrm{~s} \mathrm{[9]}$ & $1.02 \mathrm{~s}$ & $0.98 \mathrm{~s}$ \\
Simulated $t_{M B P}$ & $9.42 \mathrm{~s}$ & $0.86 \mathrm{~s}$ & $0.83 \mathrm{~s}$ \\
Measured $r_{s}$ & $0.41 \%$ & $0.27 \%$ & $0.62 \%$ \\
Simulated $r_{s}$ & (a) $0.14 \%$-(b) $1.85 \%$ & (b) $0.24 \%$ & (b) $0.79 \%$ \\
\hline
\end{tabular}

Table 2. Median block propagation time $\left(t_{M B P}\right.$, in seconds), and $r_{s}$ in the real networks and the simulation

- Impact of block interval

\begin{tabular}{ccccccccccccccccc}
\hline \multicolumn{1}{c}{ Case 1 } & \multicolumn{1}{c}{ Case 2 } & \multicolumn{1}{c}{ Case 3 } \\
\hline Block interval & $t_{M B P}$ & $r_{s}$ & $v_{d}$ & $r_{\text {rel }}$ & $t_{M B P}$ & $r_{s}$ & $v_{d}$ & $r_{\text {rel }}$ & $t_{M B P}$ & $r_{s}$ & $v_{d}$ & $r_{\text {rel }}$ & $t_{M B P}$ & $r_{s}$ & $v_{d}$ & $r_{\text {rel }}$ \\
\hline 25 minutes & 35.73 & $1.72 \%$ & 12.47 & 0.34 & 25.66 & $0.16 \%$ & 12.86 & 0.33 & 22.50 & $0.03 \%$ & 12.89 & 0.33 & 22.44 & $0.02 \%$ & 12.89 & 0.33 \\
10 minutes & 14.7 & $1.51 \%$ & 12.52 & 0.34 & 10.65 & $0.13 \%$ & 12.88 & 0.33 & 9.41 & $0.14 \%$ & 12.86 & 0.33 & 9.18 & $0.13 \%$ & 12.87 & 0.33 \\
2.5 minutes & 4.18 & $1.82 \%$ & 12.45 & 0.34 & 2.91 & $0.16 \%$ & 12.86 & 0.33 & 2.60 & $0.16 \%$ & 12.86 & 0.33 & 2.59 & $0.15 \%$ & 12.86 & 0.33 \\
1 minute & 2.08 & $2.15 \%$ & 12.35 & 0.34 & 1.34 & $0.35 \%$ & 12.81 & 0.33 & 1.30 & $0.25 \%$ & 12.83 & 0.33 & 1.27 & $0.29 \%$ & 12.77 & 0.33 \\
30 seconds & 1.43 & $2.54 \%$ & 12.06 & 0.34 & 0.84 & $0.45 \%$ & 12.78 & 0.33 & 0.84 & $0.51 \%$ & 12.77 & 0.33 & 0.84 & $0.52 \%$ & 12.69 & 0.33 \\
20 seconds & 1.21 & $3.20 \%$ & 11.73 & 0.34 & 0.67 & $0.86 \%$ & 12.68 & 0.33 & 0.69 & $0.85 \%$ & 12.68 & 0.33 & 0.68 & $0.82 \%$ & 12.68 & 0.33 \\
10 seconds & 1.00 & $4.77 \%$ & 10.73 & 0.35 & 0.35 & $1.73 \%$ & 12.46 & 0.34 & 0.33 & $1.41 \%$ & 12.54 & 0.34 & 0.53 & $1.59 \%$ & 12.50 & 0.34 \\
5 seconds & 0.89 & $8.64 \%$ & 10.08 & 0.37 & 0.37 & $2.94 \%$ & 11.85 & 0.34 & 0.45 & $2.99 \%$ & 11.80 & 0.34 & 0.44 & $3.05 \%$ & 11.78 & 0.34 \\
2 seconds & 0.84 & $16.65 \%$ & 7.35 & 0.41 & 0.40 & $6.98 \%$ & 10.47 & 0.36 & 0.39 & $7.28 \%$ & 10.37 & 0.36 & 0.38 & $7.10 \%$ & 10.42 & 0.36 \\
1 seconds & 0.82 & $26.74 \%$ & 4.37 & 0.53 & 0.53 & $12.44 \%$ & 8.34 & 0.39 & 0.38 & $12.59 \%$ & 8.24 & 0.39 & 0.37 & $12.52 \%$ & 8.30 & 0.39 \\
0.5 seconds & 0.82 & $38.15 \%$ & 2.78 & 0.60 & 0.61 & $20.62 \%$ & 6.22 & 0.42 & 0.49 & $20.87 \%$ & 6.16 & 0.42 & 0.36 & $21.10 \%$ & 6.02 & 0.42 \\
\hline
\end{tabular}

Table 3. Impact of the block interval on the median block propagation time ( $\left.t_{M B P}\right)$ in seconds, and the stale block rate $r_{s}, v_{d}$ and $r_{r e l}$

In table 3, the simulator run for different block interval times ranging from 25 minutes to 0.5 seconds. Each simulation is run independently for 10000 consecutive blocks, and for each of the four different block request management system combinations: (Case 1) the standard block request management, (Case 2) the standard block request management enhanced by unsolicited block push from the miners, (Case 3) both former components plus the relay network, and (Case 4 ) the send headers mechanism with unsolicited block push and the relay network. The impact of the block interval on the security of PoW blockchains, the results 
show that, for an adversary equipped with $30 \%$ of the total mining power, the lower is the consensus time, the higher is the relative revenue from selfish mining and the lower is the double-spending value.

- Impact of block size

\begin{tabular}{|c|c|c|c|c|c|c|c|c|c|c|c|c|c|c|c|c|}
\hline \multirow[b]{2}{*}{ Block Size } & \multicolumn{4}{|c|}{ Case 1} & \multicolumn{4}{|c|}{ Case 2} & \multicolumn{4}{|c|}{ Case 3} & \multicolumn{4}{|c|}{ Case 4} \\
\hline & $t_{M B P}$ & $r_{s}$ & $v_{d}$ & $r_{\text {rel }}$ & $t_{M B P}$ & $r_{s}$ & $v_{d}$ & $r_{\text {rel }}$ & $t_{M B P}$ & $r_{s}$ & $v_{d}$ & $r_{\text {rel }}$ & $t_{M B P}$ & $r_{s}$ & $v_{d}$ & $r_{\text {rel }}$ \\
\hline $0.1 \mathrm{MB}$ & 3.18 & $0.32 \%$ & 12.80 & 0.33 & 2.12 & $0.03 \%$ & 12.89 & 0.33 & 2.02 & $0.03 \%$ & 12.89 & 0.33 & 2.02 & $0.2 \%$ & 12.90 & 0.33 \\
\hline $0.25 \mathrm{MB}$ & 7.03 & $0.88 \%$ & 12.67 & 0.33 & 4.93 & $0.11 \%$ & 12.87 & 0.33 & 4.49 & $0.05 \%$ & 12.88 & 0.33 & 4.46 & $0.17 \%$ & 12.87 & 0.33 \\
\hline $0.5 \mathrm{MB}$ & 13.62 & $1.63 \%$ & 12.48 & 0.34 & 9.84 & $0.13 \%$ & 12.87 & 0.33 & 8.65 & $0.05 \%$ & 12.88 & 0.33 & 8.64 & $0.06 \%$ & 12.87 & 0.33 \\
\hline $1 \mathrm{MB}$ & 27.67 & $3.17 \%$ & 11.79 & 0.34 & 20.01 & $0.38 \%$ & 12.79 & 0.33 & 17.24 & $0.07 \%$ & 12.88 & 0.33 & 17.14 & $0.07 \%$ & 12.88 & 0.33 \\
\hline $2 \mathrm{MB}$ & 57.79 & $6.24 \%$ & 10.57 & 0.36 & 44.6 & $1.12 \%$ & 12.61 & 0.34 & 35.49 & $0.08 \%$ & 12.87 & 0.33 & 35.38 & $0.1 \%$ & 12.86 & 0.33 \\
\hline $4 \mathrm{MB}$ & 133.30 & $11.85 \%$ & 8.20 & 0.38 & 126.57 & $5.46 \%$ & 10.51 & 0.35 & 78.01 & $0.12 \%$ & 12.85 & 0.33 & 78.40 & $0.13 \%$ & 12.66 & 0.33 \\
\hline $8 \mathrm{MB}$ & 571.50 & $29.97 \%$ & 4.11 & 0.53 & 875.97 & $15.64 \%$ & 7.64 & 0.41 & 555.49 & $0.43 \%$ & 12.65 & 0.33 & 550.25 & $0.4 \%$ & 12.68 & 0.33 \\
\hline
\end{tabular}

Table 4. Impact of the block size on the median block propagation time $(t M B P)$ in seconds, and the stale block rate $r_{s}, v_{d}$ and $r_{r e l}$

The results from table 4 suggest that the block propagation time increases linearly with the block size up to $4 \mathrm{MB}$; after $8 \mathrm{MB}$ blocks, the block propagation time and stale block rate increases exponentially. Further, a better block propagation mechanism significantly reduces the propagation times and the stale block rate.

- Throughput

In table 9, the result shows therefore suggest that PoW blockchains can attain an effective throughput above 60 transactions per second (tps) (which implies that the current throughput of Bitcoin of 7 tps can be substantially increased) without compromising the security of the system.

\begin{tabular}{lllll}
\hline tps & $v_{d}$ & $r_{\text {rel }}$ & Block size & Block interval \\
\hline 33.4 & 12.75 & 0.33 & $0.25 \mathrm{MB}$ & 30 seconds \\
40 & 12.38 & 0.34 & $0.10 \mathrm{MB}$ & 10 seconds \\
50 & 12.45 & 0.34 & $0.25 \mathrm{MB}$ & 20 seconds \\
66.7 & 12.06 & 0.34 & $0.25 \mathrm{MB}$ & 15 seconds \\
66.7 & 12.65 & 0.33 & $0.50 \mathrm{MB}$ & 30 seconds \\
66.7 & 12.71 & 0.33 & $1.00 \mathrm{MB}$ & 1 minute \\
\hline
\end{tabular}

Table 5. Impact of the block size on the median block propagation time ( MBP $)$ in seconds, and the stale block rate $r s, v d$ and $r_{r e l}$

\section{PRIVATE BLOCKCHAIN PLATFORMS}

In A typical blockchain system consists of multiple nodes which do not fully trust each other. Some nodes exhibit Byzantine behavior, but the majority is honest. Together, the nodes maintain a set of shared, global states and perform transactions modifying the states. All nodes in the system agree on the transactions and their order 
as stored on the blockchain. Because of this, blockchain is often referred to as a distributed ledger. As a result, permissioned blockchains can execute complex application more efficiently than PoW-based blockchains, while being Byzantine fault tolerant. These properties and the commercial interests from major banking and financial institutions have bestowed on private blockchains the potentials to disrupt the current practice in data management.

\subsection{Contributions of The Paper}

- BlockBench as an evaluation framework

For understanding and comparing the performance of permissioned blockchain systems. Any private blockchain can be integrated to BlockBench via simple APIs and benchmarked against workloads that are based on real and synthetic smart contracts.

- Develop BlockBench simulator for private blockchain platform

To conduct a comprehensive evaluation of Ethereum, Parity and Hyperledger empirical results present concrete evidence of blockchain's limitations in handling data processing workloads, and reveal bottlenecks in the three systems. The results serve as a baseline for further development of blockchain technologies.

\subsection{BlockBench Simulator Implementation}

BlockBench is the first benchmarking framework for private blockchain systems. It serves as a fair means of comparison for different platforms and enables deeper understanding of different system design choices. BlockBench comes with both macro benchmark workloads for evaluating the overall performance and micro benchmark workloads for evaluating performance of individual layers.

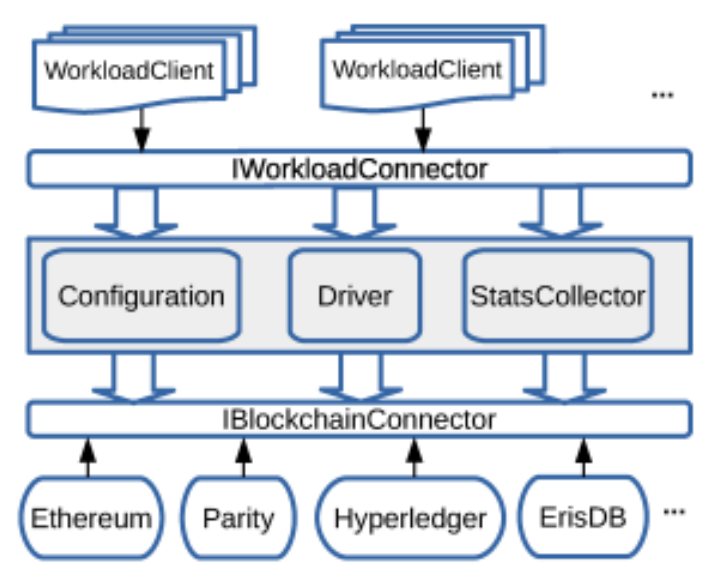

Figure 2. BlockBench software stack 
BlockBench stack consists of a frontend interface for integrating new benchmark workloads, a backend interface for integrating new blockchains, and a driver for driving the workloads. A new blockchain can be integrated into the framework's backend by implementing the IBlockchainConnector interface. Ethereum, Parity and Hyperledger are the current backends, while ErisDB (or Monax) integration are under development. A new benchmarking workload can be added by implementing IWorkloadConnector interface. The Driver takes as input a workload and sends transactions to the blockchain according to user-defined configurations. It collects runtime statistics which are used to compute five important metrics, i.e. throughput, latency, scalability, fault tolerance and security matics.

\subsection{Performance Analysis}

The author [2] selected Ethereum, Parity and Hyperledger for a comparative study using BlockBench.Also, they used the popular Go implementation of Ethereum, geth v1.4.18, the Parity release v1.6.0. Unless otherwise specified, the Hyperledger version is v0.6.0-preview. Next, set up a private testnet for Ethereum and Parity by defining a genesis block and directly adding peers to the miner network. The experiments were run on a 48-node commodity cluster. Each node has an E5-1650 3.5 GHz CPU, 32 GB RAM, 2 TB hard drive, running Ubuntu 14.04 Trusty, and connected to the other nodes via $1 \mathrm{~GB}$ switch. For Ethereum, they reserved 8 cores out of the available 12 cores per machine, so that the periodic polls from the client's driver process do not interfere with the mining process.

The main findings are as follows:

- Hyperledger performs consistently better than Ethereum and Parity across the benchmarks. But it fails to scale up to more than 16 nodes.

- Ethereum and Parity are more resilient to node failures, but they are vulnerable to security attacks that forks the blockchain.

- The main bottlenecks in Hyperledger and Ethereum are the consensus protocols, but for Parity the bottleneck is caused by transaction signing.

- Ethereum and Parity incur large overheads in terms of memory and disk usage. Their execution engine is also less efficient than that of Hyperledger.

- Hyperledger's data model is low level, but its flexibility enables customized optimization for analytical queries

To put the context of performance, they [2] compare the three blockchains against a popular in-memory database system, namely $\mathrm{H}$-Store, using the YCSB and Smallbank workload. The comparison offers useful insights into the design tradeoffs and relative performance of the two systems 


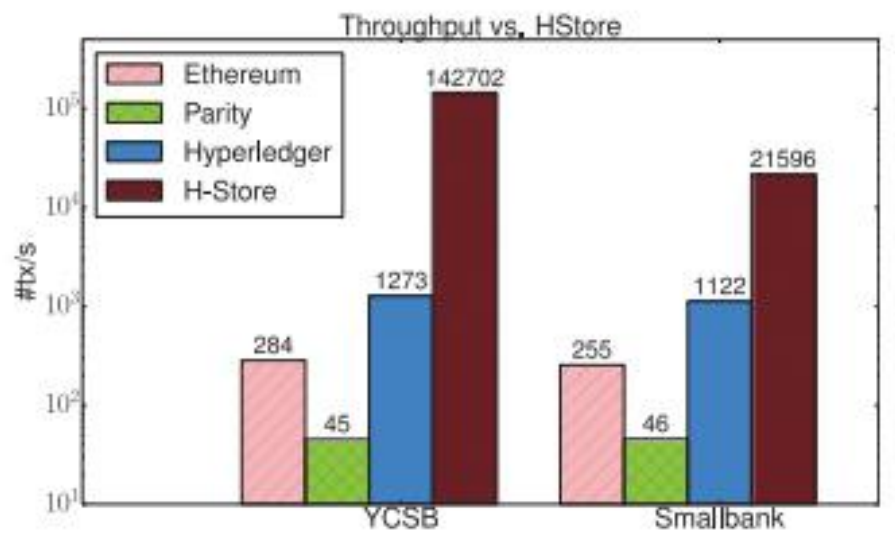

Figure 3. Performance of three blockchain system versus $\mathrm{H}$-store

Figure 3 shows at least an order of magnitude gap in throughput and two order of magnitude in latency. Specifically, $\mathrm{H}$-Store achieves over $140 \mathrm{~K}$ tx/s throughput while maintaining sub-millisecond latency. The gap in performance is due to the cost of consensus protocols. Therefore current blockchains are not well suited for large scale data processing workloads. Author demonstrated several bottlenecks and design trade-offs at different layers of the software stack

\section{CONCLUSION}

While there is a broad interest in developing blockchain systems for specific use cases, there is a lack of tools to perform their evaluation. Current evaluations are often based on emulation, which imitates the behaviour of a system in a large set of machines. This approach, however, incurs a large overhead and lacks scalability for real world deployments. Besides, power consumption of a large-scale system may have to be taken into account. An alternative is simulation. Network and distributed system simulators are important tools to evaluate the performance of protocols and systems in a large set of conditions. Simulators provide an environment that simplifies the implementation and deployment of protocols. With simulation it is possible to study a largescale system with thousands of nodes in a single machine and gather results in reasonable time.

Bitcoin simulators created to perform evaluation on the impact of network-layer parameters on the security of Bitcoin PoW. As an evaluation of the simulator, it was designed mainly to evaluate its durability against double spoofing attack when modifying the block size and block generation interval. Therefore, changing the parameters such as block size is easy. However, altering the consensus algorithm of the node or the algorithm related to the network topology is challenging. Moreover, given that many of the actual blockchain protocols are reproduced, to change the algorithm of the node, adding all the algorithms that correspond to the existing protocol is necessary. 
BlockBench is a tool to study the performance of private blockchains, to study and comparing the performance of Hyperledger Fabric, Ethereum and Parity. They found that the version of Fabric they studied did not scale beyond 16 nodes due to congestion in the message channel. The results demonstrate that these systems are still far from displacing current database systems in traditional data processing workloads. Furthermore, there are gaps in performance among the three systems which are attributed to the design choices at different layers of the blockchain's software stack.

\section{REFERENCES}

[1] A. Gervais, G. O. Karame, K. Wust, V. Glykantzis, H. Ritzdorf, and S. Capkun, "On the security and performance of Proof of Work blockchains," in Proceedings of the 2016 ACM SIGSAC Conference on Computer and Communications Security. ACM, 2016, pp. 3-16.

[2] T. T. A. Dinh, J. Wang, G. Chen, R. Liu, B. C. Ooi, and K.-L. Tan, "Blockbench: A framework for analyzing private blockchains," in Proceedings of the 2017 ACM International Conference on Management of Data. ACM, 2017, pp. 1085-1100 